\title{
MORTALIDADE POR CÂNCER DE MAMA EM MULHERES COM IDADE INFERIOR A 40 ANOS*
}

Crislayne Keretch Sebastião', Liliana Müller Larocca², Rosa Helena Silva Souza ${ }^{3}$, Marília Daniella Machado Araújo Cavalcante ${ }^{4}, V$ Viviane Serra Melanda ${ }^{5}$

Enfermeira. Técnica em Radiologia Médica. Hospital Erasto Gaertner. Curitiba-PR-Brasil. ${ }^{2}$ Enfermeira. Doutora em Educação. Universidade Federal do Paraná. Curitiba-PR-Brasil.

${ }^{3}$ Enfermeira. Mestre em Enfermagem. Doutoranda em Medicina Interna. Universidade Federal do Paraná. Curitiba-PR-Brasil.

${ }^{4}$ Enfermeira. Mestranda em Enfermagem. Universidade Federal do Paraná. Guarapuava-PR-Brasil.

${ }^{5}$ Enfermeira. Mestranda em Enfermagem. Universidade Federal do Paraná. Curitiba-PR-Brasil.

RESUMO: Trata-se de uma pesquisa documental descritiva cujo objetivo foi reconhecer o perfil epidemiológico dos óbitos ocorridos entre mulheres com menos de 40 anos, diagnosticadas com câncer de mama. A coleta dos dados foi realizada no Serviço de Registro Hospitalar de Câncer do Hospital de Clínicas da Universidade Federal do Paraná-Brasil, compreendendo informações registradas entre janeiro de 2003 e dezembro de 2011. Foram identificadas 638 notificações de câncer de mama, dos quais 68 foram de mulheres com menos de 40 anos e dessas 21 foram a óbito. Verificou-se que a sobrevida foi, em média, de 40 meses e 76,2\% apresentaram estádio clínico avançado (III ou IV). Ressalta-se que o rastreamento do câncer de mama em mulheres de alto risco, permite detectar o tumor mais precocemente, reduzir a incidência no número de óbitos e melhorar os indicadores de saúde.

DESCRITORES: Neoplasias da mama; Registros hospitalares; Enfermagem.

\section{MORTALITY FROM BREAST CANCER AMONG WOMEN BELOW 40 YEARS OLD}

\begin{abstract}
This is a descriptive documental study aiming to investigate the epidemiological profile of the deaths which occurred among women aged below 40 years old, diagnosed with breast cancer. Data collection was undertaken in the Hospital Records (Cancer) Service of the Teaching Hospital of the Federal University of Paraná, Brazil, including information recorded between January 2003 and December 2011. A total of 638 notifications of breast cancer were identified, of which 68 were of women aged below 40 years old, of whom 21 died. It was ascertained that the survival was, on average, of 40 months; and that $76.2 \%$ were at advanced stages (III or IV). It is emphasized that screening for breast cancer in women at high risk allows the detection of tumors at earlier stages, reducing the incidence in the number of deaths and improving the health indicators.
\end{abstract}

DESCRIPTORS: Breast neoplasias; Hospital records; Nursing.

\section{MORTALIDAD POR CÁNCER DE MAMA EN MUJERES CON EDAD INFERIOR A 40 AÑOS}

RESUMEN: Es una investigación documental descriptiva cuyo objetivo fue reconocer el perfil epidemiológico de los óbitos ocurridos entre mujeres con menos de 40 años, diagnosticadas con cáncer de mama. Los datos fueron obtenidos en el Servicio de Registro Hospitalar de Cáncer del Hospital de Clínicas de la Universidad Federal de Paraná-Brasil, comprendiendo informaciones registradas entre enero de 2003 y diciembre de 2011. Fueron identificadas 638 notificaciones de cáncer de mama, de las cuales 68 fueron de mujeres con menos de 40 años y de esas 21 fueron a óbito. Se verificó que la sobrevida fue, en media, de 40 meses y $76,2 \%$ presentaron estadio clínico avanzado (III o IV). Se resalta que rastrear el cáncer de mama en mujeres de alto riesgo posibilita detectar el tumor más precozmente, reducir la incidencia en el número de óbitos y mejorar los indicadores de salud. DESCRIPTORES: Neoplasias de mama; Registros hospitalares; Enfermería.

*Artigo originado da Monografia intitulada Mortalidade por câncer de mama em mulheres com idade inferior a 40 anos usuárias de um Hospital Escola de Curitiba/PR apresentada ao Curso de Graduação em Enfermagem da Universidade Federal do Paraná, 2012.

Autor Correspondente:

Marília Daniella Machado Araújo Cavalcante

Universidade Federal do Paraná

Av. Pref. Moacir Júlio Silvestri, 644 - 85010-090 Guarapuava-PR-Brasil

E-mail: profmarilia@live.com
Recebido: $29 / 10 / 2013$

Finalizado: 06/05/2014 


\section{INTRODUÇÃO}

Com as mudanças no perfil sociodemográfico da população mundial e brasileira que consequentemente alteraram o perfil epidemiológico, observa-se mudança significativa na morbimortalidade das doenças e agravos, em que as Doenças Infecciosas Transmissíveis (principal causa de morte até meados do século $X X)$ foram reduzidas e as Doenças Crônicas não Transmissíveis tiveram aumento representativo a partir da década de 1960. É possível reconhecer fatores que contribuíram para que ocorresse essa transição epidemiológica, como a diminuição da natalidade, alteração dos hábitos alimentares, aumento da expectativa de vida e sua determinação na frequência das doenças crônico-degenerativas (diabetes, doenças cardiovasculares, câncer e doenças respiratórias) ${ }^{(1)}$.

As Doenças Crônicas não Transmissíveis ganharam importância no cenário da saúde mundial, pois provocam perda de qualidade de vida, limitações nas atividades diárias, além de ser causa de elevado índice de mortalidade. No Brasil, esse grupo de doenças concentra $72 \%$ do total de óbitos, segundo dados de 2009 do Sistema de Informação de Mortalidade; este percentual representa mais de 742 mil mortes por ano. As que mais matam são as doenças cardiovasculares $(31,3 \%)$, o câncer $(16,2 \%)$, as doenças respiratórias crônicas $(5,8 \%)$ e o diabetes mellitus $(5,2 \%)$. Tal impacto pode ser revertido com medidas estruturais, inclusão social, reconhecimento de vulnerabilidades, bem como de promoção à saúde, além de atenção à saúde universalizada(2)

As neoplasias malignas ganham destaque na epidemiologia mundial, pois tem alto índice de incidência, prevalência e mortalidade, bem como elevado custo social ${ }^{(3)}$. Além disso, o tumor maligno provoca impacto em toda a família principalmente se a pessoa que adoece é a única ou principal fonte de renda familiar. Estudos realizados em parceria com a Organização PanAmericana de Saúde e o Ministério da Saúde apresentam estimativa mundial para o ano de 2030 de 27 milhões de novos casos, 17 milhões de mortes e 75 milhões de pessoas convivendo com a doença ${ }^{(4)}$.

No Brasil, de acordo com o Instituto Nacional de Câncer, órgão do Ministério da Saúde responsável pela coordenação da Política Nacional de Atenção Oncológica, há estimativa de incidência para o ano de 2013 de 518.510 novos casos de câncer. No sexo masculino serão esperados 257.870 casos novos sendo os cânceres de pele não melanoma, próstata, pulmão, cólon e reto e estômago os mais incidentes nesse grupo. Já nas mulheres a estimativa é de 260.640 novos casos principalmente de cânceres de pele não melanoma, mama, colo do útero, cólon e reto e glândula tireóide ${ }^{(4)}$.

Em todo o mundo, o câncer de mama é o segundo que mais acomete as mulheres. Dentre os fatores de risco encontra-se: idade (incidência dos casos aumenta até os 50 anos e diminui após essa idade); história familiar de câncer de mama; menarca precoce; menopausa tardia; terapia de reposição hormonal; nuliparidade; idade da primeira gestação a termo acima dos 30 anos; uso de anticoncepcionais e exposição à radiação ionizante mesmo em pequenas quantidades (principalmente durante a puberdade). Medidas como realização de atividade física, alimentação saudável e amamentação promovem menor risco de desenvolvimento de neoplasia maligna da mama ${ }^{(4)}$.

O tumor maligno de mama é o segundo tipo mais frequente nas mulheres, com estimativa de 53 mil novos casos em 2013, ficando atrás apenas dos tumores de pele não melanoma que terão incidência de 71 mil nesse grupo populacional. No Estado do Paraná, há uma estimativa de 3110 novos casos de câncer de mama no ano de 2013 (taxa bruta de incidência de 55,83 por 100 mil mulheres), desses casos, 730 só na capital do estado, Curitiba, com uma taxa bruta de 75,74 novos casos por 100 mil habitantes mulheres ${ }^{(4)}$.

Frente a tais perspectivas, justifica-se o interesse em compreender a determinação social dos óbitos por câncer de mama em mulheres jovens (com menos de 40 anos), visto que é o segundo tipo de câncer que mais acomete as mulheres em todo o mundo. Deste modo, o presente estudo tem como questão norteadora: Qual a realidade objetiva dos óbitos das mulheres com menos de 40 anos, diagnosticadas com câncer de mama, na instituição cenário da pesquisa? Acredita-se que este reconhecimento contribuirá em ações de promoção da saúde e prevenção do agravo investigado.

Este estudo tem como objetivo geral reconhecer o perfil epidemiológico dos óbitos ocorridos entre mulheres com menos de 40 anos, diagnosticadas com câncer de mama no 
Hospital de Clínicas da Universidade Federal do Paraná-Brasil. Como objetivo específico: descrever as dimensões particular e singular dos casos investigados, mediante a estrutura proposta pela Teoria da Intervenção Prática em Saúde Coletiva - TIPESC(5).

\section{MÉTODO}

Trata-se de uma pesquisa documental descritiva que foi desenvolvida no Serviço de Epidemiologia Hospitalar do Hospital de Clínicas da Universidade Federal do Paraná. A coleta dos dados foi realizada no período de 06 a 29 de maio de 2013, por meio do banco de dados do Registro Hospitalar de Câncer, com informações registradas entre janeiro de 2003 e dezembro de 2011, bem como pela consulta em prontuários e informações do Sistema de Informação Hospitalar.

Como critérios de inclusão foram selecionados notificações de casos de câncer de mama em mulheres com idade inferior a 40 anos, cuja primeira consulta aconteceu entre janeiro de 2003 e dezembro de 2011. Dentre os casos selecionados, realizou-se uma amostragem intencional, na qual foram averiguados, separadamente, os prontuários das usuárias que evoluíram para óbito.

A análise qualitativa dos dados foi inspirada nas Bases Metodológicas para a Assistência de Enfermagem em Saúde Coletiva fundamentadas no Materialismo Histórico e Dialético(5). Neste estudo foram categorizadas as dimensões particular e singular. Como referencial de análise para a dimensão particular, destacou-se o grau de instrução e a ocupação das pacientes com menos de 40 anos, diagnosticadas com câncer de mama e que evoluíram para óbito.

Na dimensão singular foram destacados: a idade das pacientes, procedência, tempo de sobrevida, estadiamento clínico, tipo histológico dos tumores, tratamento recebido, estado da doença após o primeiro tratamento, lateralidade do tumor, histórico de morte familiar de câncer e se houve orientação registrada em prontuário da paciente, para que seus familiares do sexo feminino realizassem o rastreamento de câncer de mama precocemente.

Este estudo faz parte do projeto intitulado "Promoção da saúde e prevenção de agravos no âmbito da epidemiologia hospitalar na perspectiva da saúde coletiva", com parecer de aprovação do Comitê de Ética n. 0076.0.0091.000.10.

\section{RESULTADOS}

Foram reconhecidas 638 notificações de câncer de mama realizadas entre janeiro de 2003 e dezembro de 2011, das quais 68 (10,6\%) eram mulheres com menos de 40 anos e dessas $21(30,9 \%)$ foram a óbito. Dentro da dimensão particular, com relação ao grau de instrução das 21 mulheres verificou-se que 14,3\% tinham $1^{\circ}$ grau completo, 14, 3\% concluíram o segundo grau e $4,8 \%$ possuíam ensino superior. Destacase que 33,3\% dos prontuários não apresentaram essa informação. Observou-se que 52,4\%(11 pacientes) afirmaram ter uma ocupação e 47,6\% (10 pacientes) referiram não trabalhar fora de casa.

Em relação à dimensão singular, no grupo de 21 mulheres que foram a óbito a faixa etária variou de 22 a 39 anos, sendo que $85,7 \%$ se encontravam na faixa etária de 30 a 39 anos. Observou-se, neste estudo, a predominância de mulheres oriundas do município de Curitiba $(52,4 \%)$. A sobrevida (levando-se em conta a data do diagnóstico e a data do óbito) foi em média 40 meses.

Das 68 notificações incluídas no estudo, 7,3\% apresentaram estadiamento l; 26,4\% estadiamento II; 33,8\% apresentaram estadiamento III; $13,2 \%$ o estadiamento IV e em 10 prontuários não havia essa informação. Ao analisar somente os 21 óbitos constatou-se que $80,9 \%$ das mulheres foram diagnosticadas em estadios III e IV e 14,3\% em estadio II. O tipo histológico mais frequente foi o carcinoma de células ductais $(61,9 \%)$.

Quanto aos tratamentos efetivados, 85,4\% (18 casos) das mulheres realizaram quimioterapia. Somente em dois casos (9,5\%) foi realizada a terapia com tamoxifeno (hormonioterapia) e mais outros três tipos de tratamento: cirurgia, radioterapia e quimioterapia. A maioria das que se submeteram à quimioterapia também receberam tratamento local (cirurgia e radioterapia) e apenas três casos $(14,2 \%)$ receberam tratamento sistêmico (hormonioterapia).

Em relação ao estado da doença, ao final do primeiro tratamento antineoplásico proposto, $28,6 \%$ (seis casos) encontrava-se em remissão completa, ou seja, sem evidência da doença. 
Quanto à lateralidade da mama afetada, verificouse frequência de $52,4 \%$ para a mama esquerda (11 pacientes) e 38,1\% na mama direita (oito pacientes) e apenas um caso apresentou o tumor bilateralmente.

Nesse estudo, 66,6\% (14 casos) das mulheres tinha história familiar positiva para algum tipo de câncer; dessas, seis apresentaram histórico de câncer de mama em parentes de primeiro grau. A orientação sobre a importância de que parentes de primeiro grau da paciente (filhas, irmãs) iniciassem o rastreamento antecipado para câncer de mama foi encontrada em apenas um prontuário dos 21 analisados. Uma das limitações desta constatação é o fato da informação sobre orientação ter sido averiguada somente nos prontuários; sendo que a mesma pode ter sido realizada verbalmente, porém não efetivada por meio de registro.

\section{DISCUSSÃO}

A pesquisa evidenciou que num grupo de 68 mulheres, incluídas nos critérios de inclusão do estudo e que representavam $10,6 \%$ do total de 638 casos notificados, 30,9\% foi a óbito. Estudo realizado em Ribeirão Preto mostrou que $6,5 \%$ dos casos de câncer de mama ocorreu em mulheres com menos de 40 anos e $0,6 \%$ em mulheres com menos de 30 anos $^{(6)}$.

Dentro da concepção da TIPESC considera-se que a escolaridade e a renda são determinantes para o acesso a qualquer serviço de saúde. Alguns estudos demonstraram que mulheres analfabetas têm 7,40 vezes mais chances de morrer de câncer de mama quando comparadas com as que têm nível superior; já para as com ensino fundamental completo o risco é de 3,76 vezes maior ${ }^{(7)}$. A falta de informação e o alto percentual de diagnósticos em fases tardias é um prenúncio de alta mortalidade, determinada pela baixa condição socioeconômica. As classes sociais menos favorecidas são vulneráveis, pois tem o diagnóstico tardio da doença e menor acesso a programas de prevenção ${ }^{(8)}$.

A proporção de sobrevida, quando relacionada com a escolaridade, mostra que as mulheres com nível superior têm sobrevida, em cinco anos, de $92,2 \%$. Conforme a escolaridade vai diminuindo a sobrevida, em cinco anos, também diminui: mulheres, cuja escolaridade é até o ensino médio a proporção cai para $84 \%$; nas que têm apenas o ensino fundamental fica em $73,6 \%$ e nas analfabetas em $56 \%{ }^{(9)}$. A idade também é fator de sobrevida das pacientes. Mulheres jovens, (com menos de 35 anos) ou com diagnóstico após os 75 anos têm maior risco de óbito, possivelmente porque nessas faixas etárias o câncer se apresenta de forma mais agressiva e com pior prognóstico, a melhor sobrevida se encontra na faixa etária de 40 a 49 anos $^{(8)}$. Alguns estudos apresentam taxa de mortalidade em pacientes com menos de 40 anos de 46,9\%; já nos pacientes entre 50 e 59 anos essa proporção é de $26,9 \%$. Apesar de não muito frequente os casos de câncer de mama, em mulheres com menos de 40 anos, deve ser dada especial atenção visto que a taxa de mortalidade é elevada(10).

Desde 2008 as estatísticas brasileiras apontam que cerca de $70 \%$ dos diagnósticos de câncer de mama foram realizadas em estadios tumorais avançados (III e IV) ${ }^{(8,11)}$. Com a doença mais avançada é necessária a realização de tratamentos mais radicais, o que aumenta a morbidade e provoca piora da qualidade de vida( ${ }^{(7)}$. Segundo a literatura da área, a classificação do tipo histológico é baseada nas características celulares e padrão de crescimento, sem levar em conta o local de origem da neoplasia. A maioria dos carcinomas mamários é do tipo ductal, seguido do tipo lobular. Esses dois tipos constituem juntos mais de $70 \%$ dos carcinomas. O prognóstico desfavorável é encontrado no carcinoma ductal invasivo e carcinoma lobular de formas não clássicas (variantes). Os carcinomas com prognóstico ruim são o metaplásico e o micropapilar invasivo ${ }^{(11)}$.

O câncer em sua fase inicial pode ser controlado e até mesmo curado, por meio de tratamento cirúrgico. Normalmente os tratamentos propostos associam duas ou mais abordagens terapêuticas: radioterapia, quimioterapia, hormonioterapia (tamoxifeno) para pacientes com receptores hormonais positivos, cirurgia(12). A escolha depende do estadiamento da doença e características individuais (clínicas e psicológicas) a fim de obter a melhor qualidade de vida para o paciente após os tratamentos, maiores índices de cura e menor toxicidade. Os tratamentos associados podem ser descritos quanto ao período cirúrgico como terapia neoadjuvante, se realizada antes da cirurgia ou adjuvante de realizada após a cirurgia ${ }^{(12)}$. 
Não é fácil determinar qual é e quando está concluído o primeiro tratamento visto que as intercorrências acontecem em muitos casos. Como regra geral, é considerado como primeiro tratamento aquele que foi proposto no momento do diagnóstico; caso não haja essa informação, leva-se em conta o conjunto de terapêuticas antineoplásicas aplicadas. Porém, se o paciente não tiver iniciado o tratamento em até oito meses após o diagnóstico, considera-se como tratamento não realizado, mesmo que o faça posteriormente ${ }^{(13)}$.

O termo "remissão completa da doença" é utilizado para os casos em que, após o primeiro tratamento para o câncer, o paciente não apresenta sinais da doença (o que não significa a cura). Na "remissão parcial" estão os casos em que houve resposta ao tratamento, porém o paciente continua com o tumor. A opção "doença estável" é aplicada quando o paciente recebeu o tratamento e a doença não progrediu e nem regrediu, ou seja, se manteve estacionada ${ }^{(13)}$. Quando a resposta do indivíduo ao tratamento não é satisfatória e o tumor continua a crescer, diz-se que está em "progressão". No suporte oncológico terapêutico estão enquadrados os tumores que, após realizarem o tratamento, continuam com a doença em atividade e sem recursos terapêuticos indicados para o tratamento do tumor, devendo o paciente receber apenas tratamento de suporte ${ }^{(13)}$.

Existem vários aspectos relacionados com o risco de desenvolvimento do câncer de mama, porém, o histórico familiar é um dos mais importantes. Mulheres com parentes de primeiro grau com câncer de mama têm seu risco aumentado ${ }^{(8,14)}$; este é duas vezes maior nos casos de histórico familiar de câncer de mama antes de 40 anos de idade em parentes de primeiro grau $^{(8)}$. Assim sendo, é possível indicar à equipe multiprofissional de atenção ao câncer de mama a importância de promover ações junto aos pacientes e também a seus familiares, orientando sobre a importância de que parentes de primeiro grau da paciente (filhas, irmãs) iniciem o rastreamento antecipado para câncer de mama.

\section{CONSIDERAÇÕES FINAIS}

Apesar de o câncer de mama não ser muito frequente em mulheres com menos de 40 anos, quando o mesmo ocorre, atenção especial deve ser dispensada, visto que dados epidemiológicos demonstram que o diagnóstico se faz em estadiamentos avançados, bem como há elevada taxa de mortalidade, sendo que o pior prognóstico está no grupo de mulheres com idade igual ou inferior a 35 anos.

É necessário que os profissionais de saúde reconheçam a importância do rastreamento precoce do câncer de mama em mulheres de alto risco para iniciar o tratamento tão logo se estabeleça o diagnóstico, possibilitando assim, melhoraria significativa do prognóstico, diminuição do número de óbitos e o posterior impacto nos indicadores de saúde. Cabe ao enfermeiro, além de orientar e acompanhar a paciente e seus familiares com ações individualizadas, considerando suas características pessoais e sociais, disponibilizar orientações quanto à detecção precoce do câncer de mama, especialmente ao grupo das mulheres com alto risco de desenvolver este tipo de câncer.

Espera-se com o presente estudo, contribuir com pesquisas que além de analisar o perfil epidemiológico da mortalidade das mulheres com diagnóstico de câncer de mama com idade inferior a 40 anos, oportunizem a captação da realidade objetiva deste fenômeno com a posterior elaboração de propostas de enfrentamento e intervenção, identificando vulnerabilidades e promovendo reflexões acerca da organização das políticas públicas brasileiras para o enfrentamento da DCNT.

\section{REFERÊNCIAS}

1. Lessa I, Mendonça GAS, Teixeira MTB. Doenças crônicas não transmissíveis no Brasil: dos fatores de risco ao impacto social. Bol Oficina Santi Panam. [Internet] 1996;120(5) [acesso em 21 jan 2013]. Disponível: http://hist.library.paho.org/Spanish/BOL/ v120n5p389.pdf

2. Brasil. Secretaria de Vigilância em Saúde. Departamento de Análise de Situação de Saúde. Plano de ações estratégicas para o enfrentamento das Doenças crônicas não transmissíveis (DCNT) no Brasil 20112022. [Internet] Brasília: Ministério da Saúde; 2011 [acesso em 22 jan 2013]. Disponível: http://portal. saude.gov.br/portal/arquivos/pdf/cartilha_plano.pdf

3. Aquino MMA, Coutinho KS, Marcelino JFS. Análise das notificações de câncer no município de Exu - PE entre os anos 2000 e 2007. Especialização em Gestão dos Sistemas e Serviços de Saúde. Centro de Pesquisas Aggeu Magalhães, Fundação Oswaldo Cruz, 2009 [Internet] [acesso em 21 jan 2013]. Disponível: http:// 
www.cpqam.fiocruz.br/bibpdf/2009marcelino-jfs.pdf

4. Brasil. Instituto Nacional de Câncer José Alencar Gomes da Silva (INCA). Estimativa 2012: incidência de câncer no Brasil. Coordenação Geral de Ações Estratégicas. Coordenação de Prevenção e Vigilância [Internet]. Rio de Janeiro: INCA; 2011 [acesso em 21 jan 2013]. Disponível: http://www.inca.gov.br/ estimativa/2012/estimativa20122111.pdf

5. Egry EY. Saúde coletiva: construindo um novo método em enfermagem. São Paulo: Ícone; 1996

6. Clagnan WS, Andrade JM, Carrara HHA, Tiezzi DG, Reis FJC, Marana HRC, et al. Idade como fator independente de prognóstico no câncer de mama. Rev. Bras. Ginecol. Obstet. [Internet] 2008; 30(2) [acesso em 04 jan 2014]. Disponível: http://dx.doi. org/10.1590/S0100-72032008000200004

7. Leite FMC, Bubach S, Amorim MHC, Castro DS, Primo CC. Mulheres com diagnóstico de câncer de mama em tratamento com tamoxifeno: perfil sociodemográfico e clínico. Rev. bras. cancerol. [Internet] 2011;57(1) [acesso em 05 mai 2013]. Disponível: http://www.inca.gov.br/ rbc/n_57/v01/pdf/04_artigo_mulheres_diagnostico_ cancer_mama_tratamento_tamoxifeno.pdf

8. Pinheiro AB, Lauter DS, Medeiros GC, Cardozo IR, Menezes LM, Souza RMB, et al. Câncer de mama em mulheres jovens: análise de 12.689 casos. Rev. bras. cancerol. [Internet] 2013;59(3) [acesso em 05 ago 2014]. Disponível: http://www.inca.gov.br/rbc/n_59/ v03/pdf/05-artigo-cancer-mama-mulheres-jovensanalise-casos.pdf

9. Rosa LM, Radünz V. Taxa de sobrevida na mulher com câncer de mama: estudo de revisão. Texto Contexto Enferm. [Internet] 2012;21(4) [acesso em 30 mai 2013]. Disponível: http://dx.doi.org/10.1590/S010407072012000400031

10. Lima ALP, Rolim NCOP, Gama MEA, Pestana AL, Silva EL, Cunha CLF. Rastreamento oportunístico do câncer de mama entre mulheres jovens no Estado do Maranhão, Brasil. Cad. Saúde Pública. [Internet] 2011; 27(7) [acesso em 02 jun 2013]. Disponível: http:// dx.doi.org/10.1590/S0102-311X2011000700018

11. Guimarães JRQ. Manual de oncologia. $3^{a}$ ed. São Paulo: BBS Editora; 2008.

12. Nogueira EA, Bergmann A, Paixão E, Thuler LCS. Alterações sensitivas, tratamento cirúrgico do câncer de mama e nervo intercostobraquial: revisão da literatura. Rev. bras. cancerol. [Internet] 2010;56(1) [acesso em 30 mai 2013]. Disponível: http://www.inca. gov.br/rbc/n_56/v01/pdf/12_revisao_de_literatura_ alteracoes_sensitivas_apos_cancer_mama.pdf

13. Brasil. Instituto Nacional de Câncer - INCA. Registros Hospitalares de Câncer: planejamento e gestão.
Secretaria Nacional de Assistência à Saúde. Coordenação Nacional de Controle do Tabagismo. $2^{\mathrm{a}}$ ed. Rio de Janeiro: Coordenação de Educação (CDEC); 2010.

14. Mitchell RN, Mitchell RN, Abbas AK. Fundamentos de Robbins \& Cotran: patologia. $8^{a}$ ed. Rio de Janeiro: Elsevier; 2012. 